\title{
1 Reinforcing neuron extraction and spike inference in calcium 2 imaging using deep self-supervised learning
}

3 Xinyang $\mathrm{Li}^{1,2,3 \dagger}$, Guoxun Zhang ${ }^{1,3 \dagger}$, Jiamin $\mathrm{Wu}^{1,3}$, Yuanlong Zhang ${ }^{1,3}$, Zhifeng Zhao ${ }^{1,3}$,

4 Xing Lin ${ }^{1,3,4}$, Hui Qiao ${ }^{1,3}$, Hao Xie ${ }^{1,3}$, Haoqian Wang ${ }^{2,3^{*}}$, Lu Fang $^{3,5^{*}}$ \& Qionghai Dai ${ }^{1,3^{*}}$

$5 \quad{ }^{1}$ Department of Automation, Tsinghua University, Beijing 100084, China

$6 \quad{ }^{2}$ Tsinghua Shenzhen International Graduate School, Tsinghua University, Shenzhen,

7 518055, China

$8{ }^{3}$ Institute for Brain and Cognitive Science, Tsinghua University, Beijing 100084, China.

$9{ }^{4}$ Beijing Innovation Center for Future Chips, Tsinghua University, Beijing 100084,

10 China

11 Department of Electronic Engineering, Tsinghua University, Beijing 100084, China

12 These authors contributed equally to this work.

13 *Correspondence: wanghaoqian@tsinghua.edu.cn; fanglu@tsinghua.edu.cn;

14 qhdai@mail.tsinghua.edu.cn 


\section{ABSTRACT}

Calcium imaging is inherently susceptible to detection noise especially when imaging with

high frame rate or under low excitation dosage. We developed DeepCAD, a selfand offers a new possibility for deciphering information propagation, integration, and computation in neural circuits ${ }^{5}$. To obtain accurate neuron extraction and spike inference for paucity of fluorescence photons caused by low peak accumulations and fast dynamics of in vivo calcium transients ${ }^{6,7}$, calcium imaging is easy to be contaminated by detection noise (i.e. photon shot noise and electronic noise), especially in functional imaging where high temporal resolution is particularly important for analyzing neural activities ${ }^{8}$.

To capture sufficient fluorescence photons for high-SNR calcium imaging, the most direct way is to use high excitation dosage, but concurrent photobleaching, phototoxicity ${ }^{9,10}$, and

33 tissue heating ${ }^{11}$ are detrimental for sample health and photosensitive biological processes,

34 which limits the maximal excitation power for long-term in vivo imaging ${ }^{12}$. More effective 
35 strategies include using brighter calcium indicators ${ }^{7,13}$ and more sensitive photoelectric

36 detectors ${ }^{14}$, but their performances are still largely restricted in photon-limited conditions such

37 as dendritic imaging and deep-tissue imaging. Apart from these physical or biological

38 approaches, data-driven methods are promising to offer an alternative solution to recover

39 faithful signals from degraded recordings and reduce the photon budget of calcium imaging.

40 As an intelligent signal processing technique, deep learning has been adopted by microscopists

41 and achieved impressive performance in fluorescence imaging ${ }^{15-18}$. However, calcium

42 transients are highly dynamic, non-repetitive activities and a firing pattern cannot be

without noise contamination or high-SNR images with the same underlying scene) by

extending integration time or averaging multiple noisy frames are no longer feasible, posing an entrenched obstacle for conventional supervised learning methods. 
56 time-lapse stack. We show that such a 3D self-supervised method is extremely effective for

57 calcium imaging denoising and even the subtlest calcium fluctuations induced by a single

58 action potential (AP) can be restored from severely corrupted images. Finally, a Fiji-based

59 plugin along with a pre-trained model were released to make our method easy to access and

60 convenient to use.

The general principle of DeepCAD is schematized in Fig. 1a. For network architecture, we employed $3 \mathrm{D}$ U-Net ${ }^{20}$ to aggregate spatiotemporal information in multiple frames using 3D convolutional layers (Supplementary Fig. 1, Methods), which endows DeepCAD with better denoising capability than 2D architecture or classical methods (Supplementary Fig. 2). Benefiting from the self-supervised strategy, a single low-SNR stack of $~ 3500$ frames is sufficient to be a complete training set. To generate the training set, two sub-stacks consisting of interlaced frames were split from the original low-SNR stack and 3D tiles were extracted from these sub-stacks for training (Supplementary Fig. 3). They contain approximate identical calcium transients when the original stack was imaged at near video rate, which is common for commercial or customized microscopes. After proper training, interpretable features can be

71 learned (Supplementary Fig. 4) and the model can be applied to subsequent acquisitions

72 without extra training (Fig. 1b). Although the network was trained on specified spatial and 73 temporal resolution, we found that it had non-inferior performance on various frame rates 74 (Supplementary Fig. 5) and magnifications (Supplementary Fig. 6), indicating the great scalability and generalization for versatile applications of DeepCAD. 
Notes 1-2), which contains synchronous noise-free recordings as the ground truth for comparison. The constrained nonnegative matrix factorization (CNMF) algorithm ${ }^{21}$ was correlation with the clean traces was significantly improved after denoising (Fig. 1f).

90 Even the slightest calcium transients can be restored from the original noisy data (Fig.

$91 \quad 1 \mathrm{~g}$ and Supplementary Fig. 9). These facts suggest that the spatiotemporal enhancement of DeepCAD can improve the accuracy of neuronal localization and trace extraction and largely facilitate the analysis of neural circuits. 
108 the improvement of imaging SNR, the error rate of spike inference was consequently decreased

109 (Fig. 2h and Supplementary Fig. 11). Among 107 independent calcium traces, 86\% of them

110 were observed to have lower error rates.

111 Next, we employed DeepCAD for noise removal of calcium imaging of large neuronal

112 populations in awake mice. To obtain high-SNR recordings for validation of our method, we

113 designed and built a two-photon imaging system with the capability of simultaneous low-SNR

114 and high-SNR recording (Supplementary Fig. 12 and Methods). The high-SNR detection path

115 was strictly synchronized with the low-SNR detection path but with about 10-fold higher

116 imaging SNR (Supplementary Fig. 13), which can be used as the reference for our denoising 
118 expressing GCaMP6f and found that calcium fluctuations indiscernible in original low-SNR

119 recordings can be effectively recovered by DeepCAD (Fig. 3a-c and Supplementary Video 2).

120 The imaging SNR was improved more than 10 folds considering that the SNR of enhanced

121 recordings even surpasses corresponding high-SNR reference. Fluorescence traces of dendritic

122 pixels can be accurately resolved and keep high consistency with the high-SNR reference (Fig.

123 3d-e and Supplementary Fig. 14). We also applied DeepCAD to enhance calcium imaging of

124 somatic signals. After denoising, neuronal distribution and circuit dynamics can be recognized

125 from a single frame (Fig. 3f-h and Supplementary Video 3). Using CNMF as the downstream

126 source extraction method, $52.6 \%$ (229 contrast to 150$)$ more active neurons can be extracted

127 (Fig. 3i,j and Supplementary Fig. 15) and the trace peak SNR of extracted neurons was also

128 improved more than two folds (9.9 contrast to 4.8, median value) (Fig. 3k), indicating that the

129 functional analysis of large neuronal populations can be effectively strengthened due to

130 improved SNR.

131 In summary, we demonstrate DeepCAD, a deep self-supervised learning-based method for

132 spatiotemporal enhancement of calcium imaging. Quantitative evaluation on both simulated

133 and experimental data shows that the accuracy of neuron extraction and spike inference can be

134 largely reinforced after denoising. To fully evaluate the capability and reliability of our method,

135 a customized two-photon microscope was built to capture synchronized low-SNR and high-

136 SNR recordings, which indicates that DeepCAD enables a more than tenfold improvement in

137 imaging SNR. To maximize its accessibility, we released an open-source Fiji plugin

138 (Supplementary Fig. 16 and Supplementary Notes 3) and a pre-trained DeepCAD model for 
139 two-photon imaging of neuron populations. Our method can be efficiently configured on a

140 common desktop and achieve comparable performance on different imaging systems

141 regardless of objectives and detectors (Supplementary Fig. 17 and Supplementary Video 4).

142 Although DeepCAD is currently investigated only on two-photon microscopy, it can be easily

143 extended to other imaging modalities such as wide-field microscopy and light-sheet

144 microscopy. We anticipate that this method could serve as a general processing step for calcium

145 imaging in photon-limited conditions and promote long-term and high-fidelity recording of

146 neural activities. 


\section{Methods}

148 Optical setup. A two-photon imaging system was designed to capture strictly

149 synchronized low-SNR and high-SNR calcium recordings for validation of our method.

150 Our system was based on a standard two-photon laser scanning microscope (2PLSM)

151 and the detection path was specially designed to split the fluorescence in a ratio of 1:10.

152 All components of our imaging system are commercially available or easy to fabricate.

153 The schematic of the custom-built two-photon microscope is shown in Supplementary

154 Fig. 12. At the forefront of the optical path, a titanium-sapphire laser system with tunable

155 wavelength (Mai Tai HP, Spectra-Physics) was used as the illumination source to emit

156 the linearly polarized, femtosecond-pulsed Gaussian excitation beam (920 nm central

157 wavelength, pulse width $<100$ fs, $80 \mathrm{MHz}$ repetition rate). A half-wave plate

158 (AQWP10M-980, Thorlabs) was used to adjust the polarization of the laser beam. Then

159 the laser beam went through an electro-optic modulator (350-80LA-02, Conoptics) to

160 modulate the excitation power and the half-wave plate was rotated to make the electro-

161 optic modulator have maximal extinction ratio. A 4f system composed of two

162 achromatic lenses (AC508-200-B, Thorlabs) with the same focal length was followed

163 to collimate the laser beam. Another 4f system (AC508-100-B and AC508-400-B,

164 Thorlabs) with a fourfold magnification was used to expand the laser beam and guide

165 the beam into a galvo-resonant scanner $(8315 \mathrm{~K} / \mathrm{CRS} 8 \mathrm{~K}$, Cambridge Technology) for

166 fast optical scanning. The scanner mount was optimally designed for reliable and

167 distortion-free scanning. Then the beam went through a scan lens (SL50-2P2, Thorlabs) 
168

and a tube lens (TTL200MP, Thorlabs) and converged into a tight focus through a high numerical aperture (NA) water-dipping objective (25×/1.05 NA, XLPLN25XWMP2, Olympus). A high-precision piezo actuator (P-725, Physik Instrumente) was additionally used to drive the objective for fast axial scanning. The beam size at the back aperture of the objective was further restricted with an iris set behind the beam expander (L4) to keep the back aperture of the objective underfilled. The effective excitation NA was about 0.5 in our imaging experiments.

collected by the objective. High-NA detection is helpful to detect more fluorescence photons and improve the signal intensity. A long-pass dichroic mirror (DMLP650L, Thorlabs) was used to separate fluorescence by reflecting the fluorescence signals and transmitting the excitation light. A 1:9 (reflectance: Transmission) non-polarizing plate beam splitter (BSN10, Thorlabs) was then placed in the detection path. All fluorescence going through the beam splitter will be split into a $10 \%$ component (low-SNR path) and a $90 \%$ component (high-SNR path), propagating in two orthogonal directions and detected by two photomultiplier tubes (PMT1001, Thorlabs). A pair of fluorescence filters (MF525-39, Thorlabs; ET510/80M, Chroma) was configured in front of each PMT to fully block wavelengths outside the emission passband of green fluorescent protein (GFP). To improve detection efficiency, we conjugated the back aperture of the objective to the sensor planes of the two PMTs using two 4f systems (TTL200-A and 
190 suffers from noise, it can be used as the reference to identify underlying structures and

191 calcium fluctuations. The field-of-view (FOV) of our two-photon imaging system is

192 about $600 \mu \mathrm{m}$ and the frame rate is about $30 \mathrm{~Hz}$.

193 System calibration. To confirm the fluorescence intensity ratio between the high-SNR

194 detection path and the low-SNR detection path, we imaged $1 \mu \mathrm{m}$ green-fluorescent beads (G0100, ThermoFisher) for system calibration. The beads suspension was first diluted and embedded in 1.0\% agarose and then mounted on a microscope slide to form

201 (Supplementary Fig. 13). All beads in the FOV were manually segmented and the

204 detection path was approximately tenfold higher than that of the low-SNR detection 205 path.

206 Mouse preparation and calcium imaging. All experiments involving mice were 207 performed in accordance with institutional guidelines for animal welfare and have been 208 approved by the Institutional Animal Care and Use Committee (IACUC) of Tsinghua 
211 anesthetized with $1.5 \%$ isoflurane and craniotomy surgeries were conducted using a

212 stereotaxic instrument (68018, RWD Life Science) under a bright-field binocular

213 microscope (77001S, RWD Life Science). A custom-made coverslip fitting the shape

214 of the cranial window ( $\sim 6 \mathrm{~mm}$ in diameter) was embedded and cemented to the skull.

215 A biocompatible titanium headpost was then cemented to the skull for stable head

216 fixation. The edge of the cranial window was enclosed with dental cement to hold the

217 immersion water of the objective. After the surgery, $0.25 \mathrm{mg} / \mathrm{g}$ body weight of

218 Trimethoprim (TMP) was intraperitoneally injected to induce the expression of

219 GCaMP6f genetically encoded calcium indicator (GECI) in layer 2/3 neurons across

220 the whole brain. To reduce potential inflammation, $5 \mathrm{mg} / \mathrm{kg}$ body weight of Ketoprofen

221 was injected subcutaneously. Each mouse was housed in a separate cage for 1-2 weeks

222 of postoperative recovery.

223 Imaging experiments were carried out when the cranial window became clear and

224 no inflammation occurred. Mice were first rapidly anesthetized with $3.0 \%$ isoflurane

225 and then fixed onto a custom-made holder with the headpost. The mouse holder was

226 mounted on a precision translation stage with three motorized axes (M-VP-25XA-

227 XYZL, Newport) to find the region of interest (ROI) for imaging. The correction ring

228 of the objective was adjusted to compensate for the coverslip thickness and eliminate

229 spherical aberrations. The excitation power after the objective was kept below $140 \mathrm{~mW}$

230 in all experiments to avoid potential laser-induced tissue damage. Gaseous anesthesia 
231 was switch off and the mice kept awake during the whole imaging process.

232 Network architecture and training details. The network architecture of DeepCAD

233 employs 3D U-Net, which is reported to have superior performance on the segmentation

234 of volumetric data ${ }^{20}$. In general, the network is composed of a $3 \mathrm{D}$ encoder module (the

235 contracting path), a 3D decoder module (the expanding path), and three skip

236 connections from the encoder module to the decoder module (Supplementary Fig. 1). In

237 the 3D encoder module, there are three encoder blocks. Each block consists of two

$2383 \times 3 \times 3$ convolutional layers followed by a leaky rectified linear unit (LeakyReLU) and

239 a $2 \times 2 \times 2$ max pooling with strides of two in three dimensions. In the decoder module,

240 there are three decoder blocks, each of which contains two $3 \times 3 \times 3$ convolutional layers

241 followed by a LeakyReLU and a 3D nearest interpolation. A group normalization ${ }^{22}$

242 layer is configured after each convolutional layer. The skip connections link low-level

243 features and high-level features by concatenating their feature maps. All operations

244 (convolutions, max poolings, and interpolations) in the network are in 3D to aggregate

245 spatial information and temporal information. For the loss function, we used the

246 arithmetic average of a L1-norm loss term and a L2-norm loss term. The model was

247 trained on 3D tiles with a spatial size of $64 \times 64$ pixels and a temporal size of 300 frames.

248 Small spatial size can lower memory requirements and reduce the training time, and

249 large temporal size is helpful to make full use of temporal information.

250 Adam optimizer ${ }^{23}$ was used for network training with a learning rate of 0.00005 and

251 exponential decay rates of 0.5 for the first moment and 0.9 for the second moment. We 
used Graphics Processing Units (GPU) to accelerate the training and test process. It took about 12 hours to train our model for 20 epochs on a typical training set (about 1200 3D tiles) with a single GPU (Nvidia TITAN RTX, 24 GB memory). Training time can be further shortened by using a more powerful GPU or parallelizing the training process on multiple GPUs. convolutions usually involve more parameters than the $2 \mathrm{D}$ counterpart. The best denoising performance is only achieved at the point where there is neither underfitting nor overfitting. To screen out the model with the best generalization ability, we saved

261 the network snapshot after each training epoch and evaluated its performance on a

262 holdout validation set. We fed the validation data into each model and calculated the

263 standard deviation projection of the output stack of each model. Then, the average pixel

264 intensity was calculated on a small dark region (e.g. blood vessels or a small region 265 without neural activity during the recording) of all standard deviation projections. The 266 best model was selected to be the one with the smallest dark standard deviation.

267 Data simulation. Our simulation program includes a step for synthesizing the noise-

268 free video (ground truth) and a step for adding the Mixed Poisson-Gaussian (MPG)

269 noise (Supplementary Notes 1-2). Firstly, to generate realistic simulated calcium imaging 270 data, we constructed a neuron library containing the spatial profiles of 517 neurons.

271 These neurons were extracted using the constrained nonnegative matrix factorization 272 algorithm ${ }^{21}$ (CNMF) from an experimentally obtained two-photon calcium imaging 
273 data of a virus-transfected wild-type mouse expressing GCaMP6f (layer 2/3 at the

274 primary somatosensory cortex). For the spatial component that defines the location of

275 each neuron, 120 neurons were randomly selected from the library to keep the sparsity

276 of neurons. For the temporal component that defines the fluorescence fluctuations of

277 each neuron, MLspike ${ }^{24}$ was employed to generate calcium traces with GCaMP6f

278 kinetics. Then, these two components were reshaped into 2D matrices and the simulated

279 noise-free data $(1 \mu \mathrm{m} /$ pixel spatial sampling rate, $30 \mathrm{~Hz}$ frame rate $)$ was synthesized as

280 the product of the spatial matrix and the temporal matrix. The noise-contaminated

281 counterpart was ultimately generated by adding the content-related MPG noise. Data

282 with different imaging SNRs were simulated with different relative photon numbers.

283 Their relationship was investigated in Supplementary Fig. 7. All images were saved as

284 uncompressed tif files with the format of unsigned 16-bit integer (uint16). More details

285 of data simulation and related mathematical models are described in Supplementary

286 Notes 1-2.

287 Single-neuron recordings. The data of simultaneous two-photon imaging and

288 electrophysiological recordings of single-neuron activities were released by the

289 Svoboda $\mathrm{lab}^{25}$ and were downloaded from the Collaborative Research in Computational

290 Neuroscience (CRCNS) platform. Only recordings of GCaMP6f neurons were used in

291 this study. The image stacks were fourfold downsampled to reduce the sampling rate

292 and some outlier recordings with very sparse spikes and low electrophysiological SNR

293 were excluded. Fluorescence traces were extracted from temporal stacks using 
294 manually annotated cytoplasmic masks. For spike inference, we used the MLspike

295 algorithm ${ }^{24}$, which was reported to rank first in the Spikefinder challenge ${ }^{26}$. All traces

296 were divided by their mean values for normalization before fed into the spike inference

297 pipeline. Recommended model parameters for GCaMP6f indicator were used to ensure

298 optimal performance of spike inference.

299 Data analysis of neuronal populations. Calcium imaging data of large neuronal

300 populations were first registered with a non-rigid motion correction method ${ }^{27}$ and the

301 black edges of registered images were clipped. Then, $\mathrm{CNMF}^{21}$ was employed as the

302 source extraction method for neuron segmentation and trace extraction. A spatial matrix

303 and a temporal matrix can be obtained from each video, storing the spatial footprints

304 and corresponding calcium traces of all active neurons, respectively. The same set of

305 parameters was used for the original low-SNR recording and corresponding DeepCAD

306 enhanced counterpart, as well as the high-SNR recording. Simulated data were analyzed

307 following the same pipeline except motion correction. Along with automatic neuron

308 extraction, we also performed manual annotations to inspect our results. High-SNR

309 recordings were tenfold downsampled along the time axis by averaging each

310 consecutive ten frames, which reduced the disturbance of detection noise and was

311 helpful to improve annotation accuracy. Boundaries of all active components were

312 annotated using the ROI Manager toolbox of Fiji. The final segmentation masks were

313 generated through subsequent morphological operations of images and connected

314 domain extraction implemented with customized MATLAB scripts. 
315 Performance metrics. Two types of metrics were used for quantitative evaluation of

316 the spatial and temporal performance of DeepCAD. For synthetic calcium imaging data,

317 corresponding clean images and ground-truth calcium traces were available. SNR and

318 PSNR were used as the spatial metric to evaluate pixel-level similarity between

319 DeepCAD enhanced images and ground-truth images. Pearson correlation coefficient

320 (R) was used as the temporal metric to reflect the similarity between enhanced traces

321 and ground-truth traces. The Pearson correlation between signal $x$ and the reference

322 signal $y$ is defined as

$$
R=\frac{\mathrm{E}\left[\left(x-\mu_{x}\right)\left(y-\mu_{y}\right)\right]}{\sigma_{x} \sigma_{y}}
$$

324 where $\mu_{x}$ and $\mu_{y}$ are the mean values of signal $x$ and $y$, respectively; $\sigma_{x}$ and $\sigma_{y}$ are the

325 standard deviations of signal $x$ and $y$, respectively; E represents arithmetic mean.

326 Furthermore, we also evaluated the performance of DeepCAD based on more

327 complex downstream tasks such as neuron extraction and spike inference, which are

328 the most crucial prerequisites in functional analysis of neural circuits from calcium

329 imaging data. We considered neuron extraction as an instance segmentation problem

330 and adopted an object-level metric to evaluate segmentation performance ${ }^{28}$. Different

331 intersection-over-union (IoU, defined as the intersection area divided by the union area

332 of two objects) thresholds were selected to determine correctly segmented objects. For

333 a specified IoU threshold, the segmentation accuracy (F1 score) was defined as the

334 harmonic mean of sensitivity and precision, which can be formulated as 


$$
\mathrm{F} 1=\frac{2 \mathrm{TP}}{2 \mathrm{TP}+\mathrm{FP}+\mathrm{FN}}
$$

336 Here, TP, FP, and FN are the number of true positives, false positives, and false

337 negatives, respectively. When applied CNMF as the source extraction method, the SNR

338 of calcium traces was quantified with the peak SNR automatically calculated by the

339 CaImAn toolbox ${ }^{29}$ with infinite outliers eliminated. For spike inference, we used the

340 error rate (ER) to quantify the performance of spike inference, which is defined as ER

$341=1-$ F1. Spikes detected from simultaneous electrophysiological recordings were used

342 as the ground truth for ER calculation. The evaluation process was implemented with

343 customized MATLAB scripts. SNR, PSNR, Pearson correlation coefficient, and IoU

344 were computed using built-in functions.

\section{Data availability}

346 Our data will be made publicly available post peer-review.

\section{Code availability}

348 Our python code and Fiji plugin will be made publicly available post peer-review.

\section{Acknowledgements}

350 We would like to acknowledge Y. Tang and Y. Yang at the School of Medicine of Tsinghua

351 University for providing transgenic mice for imaging and the mesoscope imaging data for

352 cross-system validation. We thank the Svoboda lab at Janelia Research Campus for releasing 
353 their data of simultaneous electrophysiology and two-photon recording. This work was

354 supported by the National Natural Science Foundation of China (62088102, 61831014, 35561531014 and 6181001011) and the Shenzhen Science and Technology Project under Grant 356 (ZDYBH201900000002 and JCYJ20180508152042002).

\section{Author Contributions}

358 Q. D., H. W., L. F. and XY. L. conceived this project. Q. D., H. W. and L. F. supervised this 359 research. XY. L. and G. Z. designed detailed implementations and processed the data. XY. L 360 designed and set up the imaging system. XY. L and G. Z. conducted the experiments. G. Z. 361 developed the python code and the Fiji plugin. J. W., Y. Z., and X. L. directed the experiments 362 and data analysis. L. F., Y. Z., Z. Z, H. Q. and H. X. gave critical support on system setup and 363 imaging procedure. J. W., L. F., Y. Z., X. L., H. Q., H. X., H. W. and Q. D. gave critical 364 discussions on the results. All authors participated in the writing of the paper. 


\section{References}

366 1. Grienberger, C. \& Konnerth, A. Imaging Calcium in Neurons. Neuron 73, 862$367 \quad 885(2012)$.

368 2. Lu, R. et al. Video-rate volumetric functional imaging of the brain at synaptic resolution. Nat. Neurosci. 20, 620-628 (2017).

3. Weisenburger, $\mathrm{S}$. et al. Volumetric $\mathrm{Ca}(2+)$ Imaging in the Mouse Brain Using Hybrid Multiplexed Sculpted Light Microscopy. Cell 177, 1050-1066 e1014 (2019).

4. Chow, D.M. et al. Deep three-photon imaging of the brain in intact adult zebrafish. Nat. Methods 17, 605-608 (2020).

5. Calarco, J.A. \& Samuel, A.D. Imaging whole nervous systems: insights into behavior from worms to fish. Nat. methods 16, 14-15 (2019).

6. Sabatini, B.L., Oertner, T.G. \& Svoboda, K. The Life Cycle of Ca2+ Ions in Dendritic Spines. Neuron 33, 439-452 (2002).

7. Chen, T.W. et al. Ultrasensitive fluorescent proteins for imaging neuronal activity. Nature 499, 295-300 (2013).

8. Ji, N., Freeman, J. \& Smith, S.L. Technologies for imaging neural activity in large volumes. Nat. Neurosci. 19, 1154-1164 (2016).

9. Svoboda, K. \& Yasuda, R. Principles of two-photon excitation microscopy and its applications to neuroscience. Neuron 50, 823-839 (2006).

10. Skylaki, S., Hilsenbeck, O. \& Schroeder, T. Challenges in long-term imaging and quantification of single-cell dynamics. Nat. Biotechnol. 34, 1137-1144 (2016).

11. Podgorski, K. \& Ranganathan, G. Brain heating induced by near-infrared lasers during multiphoton microscopy. J. Neurophysiol. 116, 1012-1023 (2016).

12. Wang, T. et al. Quantitative analysis of 1300-nm three-photon calcium imaging in the mouse brain. eLife 9 (2020).

13. Dana, H. et al. High-performance calcium sensors for imaging activity in neuronal populations and microcompartments. Nat. Methods 16, 649-657 (2019).

14. Samantaray, N., Ruo-Berchera, I., Meda, A. \& Genovese, M. Realization of the first sub-shot-noise wide field microscope. Light Sci. Appl. 6, e17005 (2017).

15. Weigert, M. et al. Content-aware image restoration: pushing the limits of fluorescence microscopy. Nat. Methods 15, 1090-1097 (2018).

16. Belthangady, C. \& Royer, L.A. Applications, promises, and pitfalls of deep learning for fluorescence image reconstruction. Nat. Methods 16, 1215-1225 (2019).

402 17. Wang, H. et al. Deep learning enables cross-modality super-resolution in fluorescence microscopy. Nat. Methods 16, 103-110 (2019). 
19. Lehtinen, J. et al. Noise2Noise: Learning Image Restoration without Clean Data. In Proc. 35th International Conference on Machine Learning (eds Dy, J. \& Krause, A.) 2965-2974 (PMLR, 2018).

20. Çiçek, Ö. et al. 3D U-Net: learning dense volumetric segmentation from sparse annotation. In Medical Image Computing and Computer-Assisted Intervention 424-432 (2016).

21. Pnevmatikakis, E.A. et al. Simultaneous Denoising, Deconvolution, and Demixing of Calcium Imaging Data. Neuron 89, 285-299 (2016).

22. Wu, Y. \& He, K. Group normalization. In 2018 IEEE European Conference on Computer Vision (ECCV) 3-19 (IEEE, 2018).

23. Kingma, D. P. \& Ba, J. L. Adam: a method for stochastic optimization. In International Conference on Learning Representations 1-15 (2015).

24. Deneux, T. et al. Accurate spike estimation from noisy calcium signals for ultrafast three-dimensional imaging of large neuronal populations in vivo. Nat. Commun. 7, 12190 (2016).

25. GENIE project, Janelia Farm Campus, HHMI; Karel Svoboda (contact). (2015). Simultaneous imaging and loose-seal cell-attached electrical recordings from neurons expressing a variety of genetically encoded calcium indicators. CRCNS.org. http://dx.doi.org/10.6080/K02R3PMN

26. Berens, P. et al. Community-based benchmarking improves spike rate inference from two-photon calcium imaging data. PLoS Comput. Biol. 14, e1006157 (2018).

27. Pnevmatikakis, E.A. \& Giovannucci, A. NoRMCorre: An online algorithm for piecewise rigid motion correction of calcium imaging data. J. Neurosci. Methods 291, 83-94 (2017).

28. Caicedo, J.C. et al. Nucleus segmentation across imaging experiments: the 2018 Data Science Bowl. Nat. Methods 16, 1247-1253 (2019).

29. Giovannucci, A. et al. CaImAn an open source tool for scalable calcium imaging data analysis. eLife 8 (2019). 

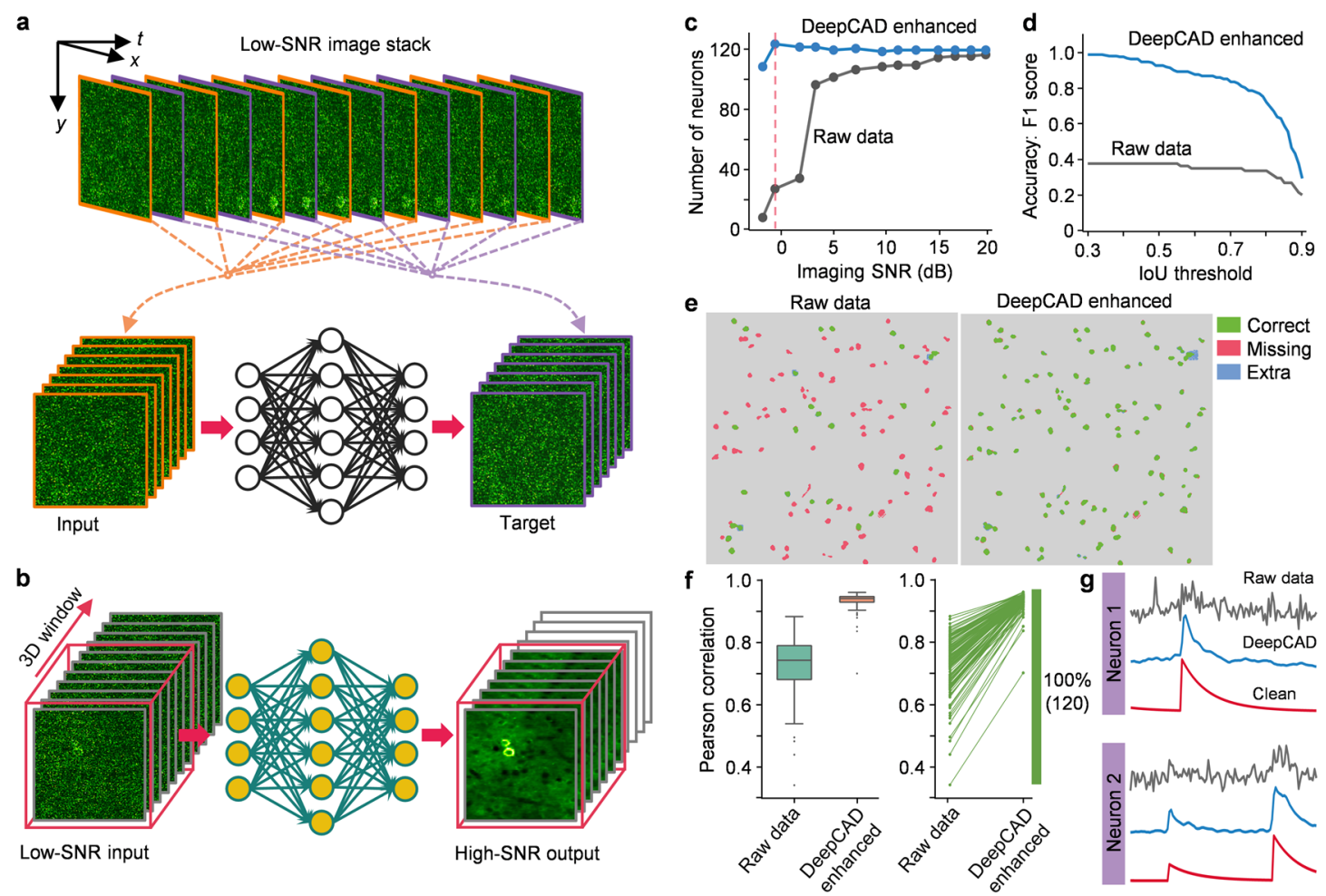

Fig. 1 | General principle and validation of DeepCAD. a, Self-supervised training strategy of DeepCAD. Consecutive frames in the original low-SNR stack are divided into two sub-stacks, used as the input volume and corresponding target volume to train a deep neural network (3D U-Net). After training, a denoising model can be established and memorized in network parameters. b, Application of the DeepCAD model. For subsequent acquisitions, a 3D $(x-y-t)$ window traverses the entire stack and 3D tiles are sequentially fed into the pre-trained model. Denoised recordings will be obtained after the processing of the model. c, The number of neurons extracted under different imaging SNRs before and after the enhancement of DeepCAD. N=120 active neurons were simulated in the field of view (FOV). d, Accuracy of neuron segmentation quantified with $\mathrm{F} 1$ score at different intersection-over-union (IoU) thresholds (imaging $\mathrm{SNR}=-0.7 \mathrm{~dB}$, indicated by the red dashed line in $\mathbf{c})$. e, Spatial profiles of extracted neurons (imaging $\mathrm{SNR}=-0.7 \mathrm{~dB}$ ). Correctly segmented regions (true positive) are colored green. Missing (false negative) and extra regions (false positive) are colored red and blue, respectively. Neuron extraction was implemented with $\mathrm{CNMF}^{21}$. f, Left:

451 boxplot showing the distribution of Pearson correlation coefficients with clean traces 452 before and after denoising $(\mathrm{N}=120)$. Right: increases of trace correlations. Each line represents one of 120 calcium traces and correlation coefficients of all neurons were observed improved. g, Calcium transients indiscernible from noise (gray) can be restored by DeepCAD (blue). Traces without noise contamination (red) serve as the ground truth for comparison. 

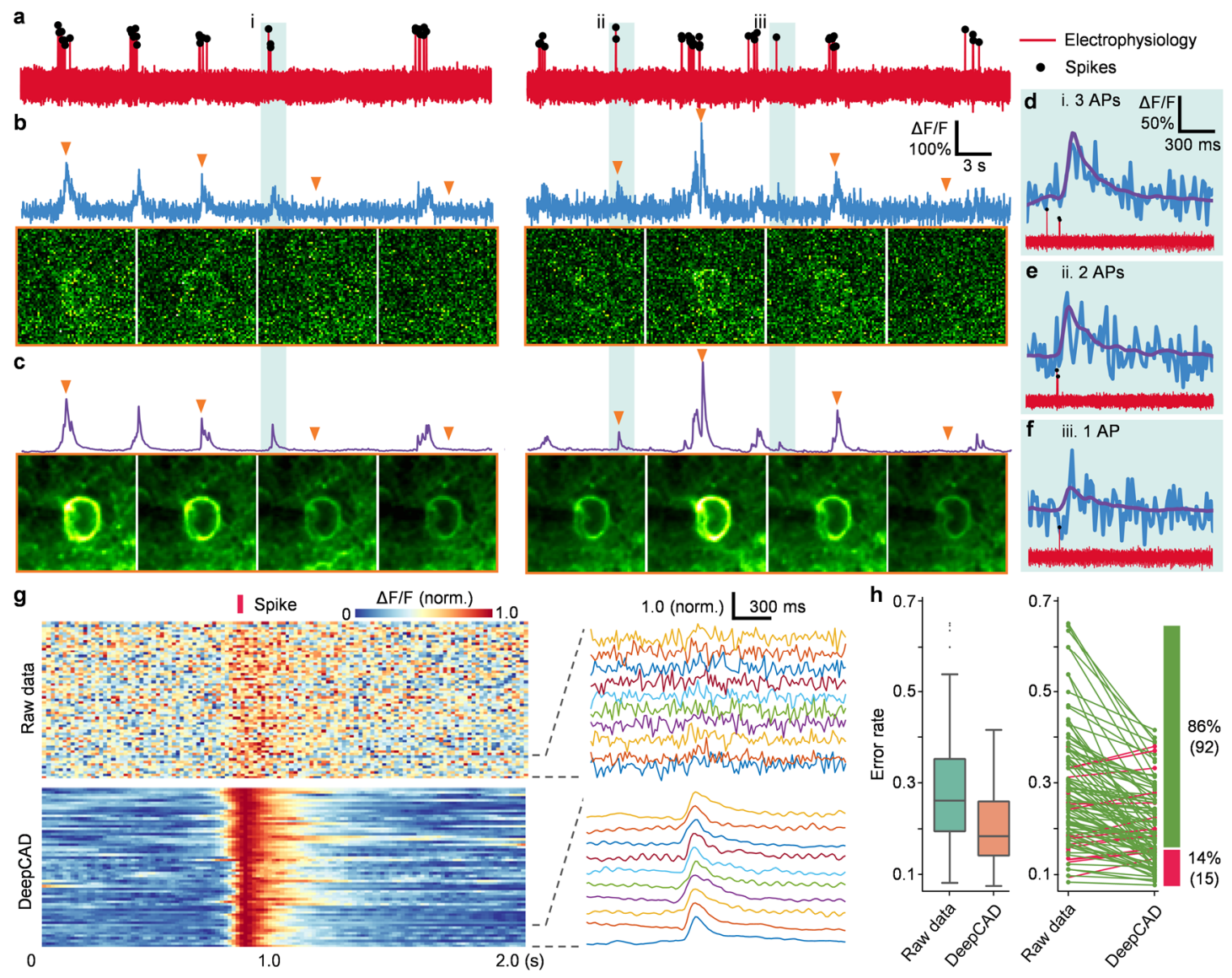

Fig. 2 | Spatiotemporal enhancement of DeepCAD. a, Electrophysiological recordings of neural activities from a single neuron. Detected spikes are marked with black dots. b, Two-photon calcium imaging of the same neuron synchronized with cellattached electrophysiology. Both spatial footprints and temporal traces of the neuron were severely corrupted in detection noise. Representative frames indicated with orange triangles are presented below the trace. $\mathbf{c}$, Fluorescence traces and representative frames after the enhancement of DeepCAD. d-f, The most imperceptible calcium transients evoked by three APs (d), two APs (e), and one AP (f) can be resolved and still keep their original dynamics noise removal. g, Calcium fluctuations evoked by 61 isolated action potentials. All spikes were normalized and temporally aligned with the red bar. Zoom-in traces are shown in the right panel. $\mathbf{h}$, Left: Boxplot showing the distribution of error rates (lower is better) of spike inference for calcium traces extracted

470 from enhanced data compared with those extracted from the original data $(\mathrm{N}=107)$.

471 Real spike timings were revealed by simultaneous cell-attached recordings. Right:

472 decreases of the error rate of spike inference. Each line represents one of 107 recordings, using green for decreased error rates and red for increased error rates. 

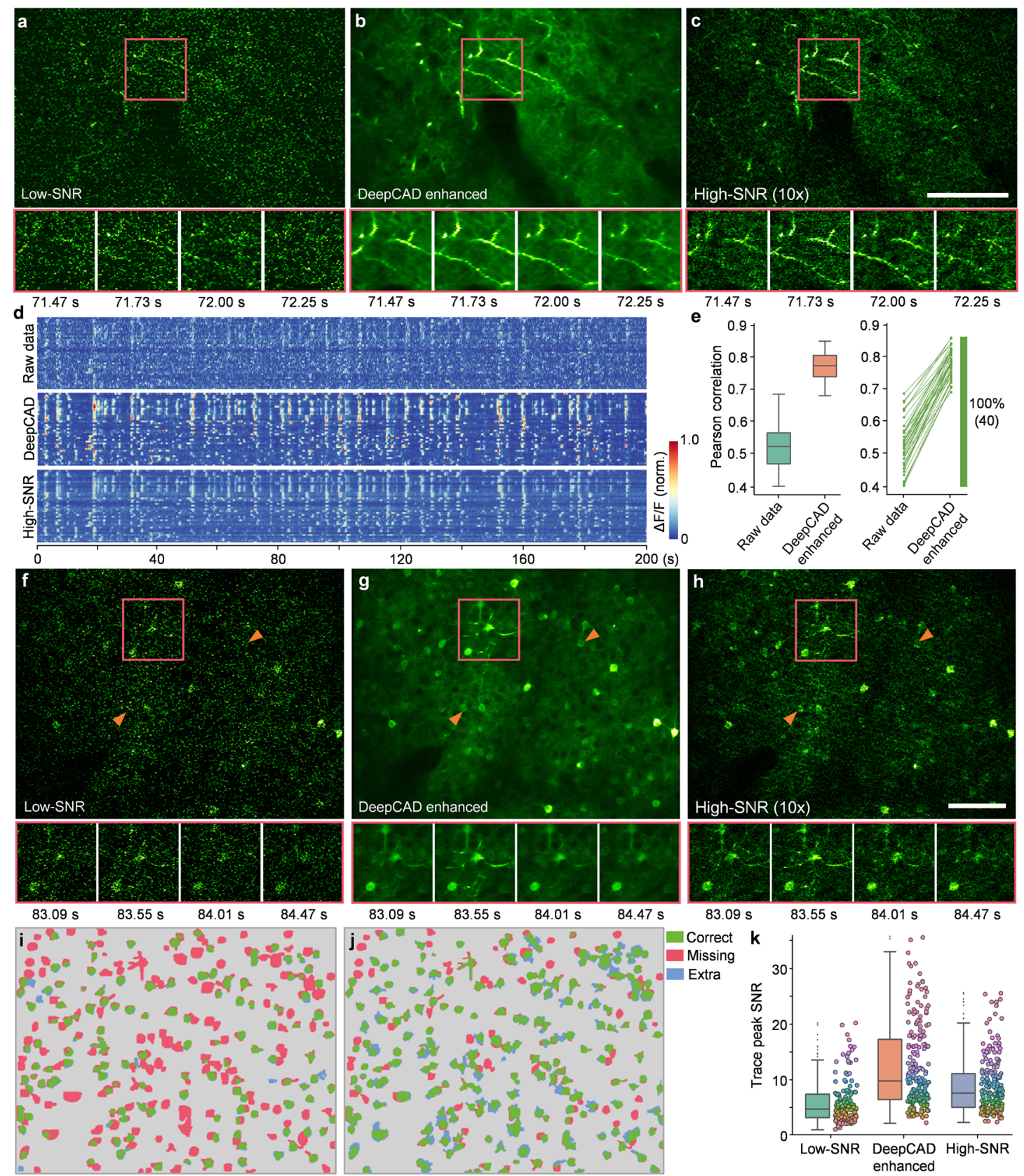

Fig. 3 | DeepCAD reinforces the recording of large neuronal populations. a, Spontaneous neuropil activities in layer 1 of the mouse cortex captured by the low-SNR detection path. b. Images restored from the low-SNR recording using DeepCAD. c,

478 Synchronized recording acquired by the high-SNR detection path (10-fold imaging

479 SNR). Magnified views of the boxed regions show calcium transients in a $\sim 0.8 \mathrm{~s}$ time 480 window. Scale bar $100 \mu \mathrm{m}$. d, Fluorescence traces extracted from 40 dendritic pixels. 481 Top: low-SNR recording, Middle: DeepCAD enhanced recording, Bottom: high-SNR 482 recording. e, Pearson correlation coefficients of single-pixel calcium traces before and 483 after denoising (left). High-SNR traces were used as the reference for correlation 484 calculation. Improvements were observed in all 40 traces (right). f, Low-SNR recording 485 of somatic signals in cortical layer $2 / 3$. g, DeepCAD enhanced recording. h, 486 Synchronized high-SNR recording (10-fold imaging SNR). Orange arrows point to two 

window. Scale bar $100 \mu \mathrm{m}$. i, Neurons extracted from the original low-SNR recording

$489(\mathrm{~N}=150)$. $\mathbf{j}$, Neurons extracted from the DeepCAD enhanced recording $(\mathrm{N}=229)$.

490 Manual annotations served as the ground truth. Correctly segmented regions (true

491 positive) are colored green. Missing (false negative) and extra regions (false positive)

492 are colored red and blue, respectively. $\mathbf{k}$, Distribution of peak SNRs of extracted

493 calcium traces. CNMF was used for source extraction and peak SNR estimation. 